OPEN ACCESS

Edited by:

Fabrizio Piras,

Santa Lucia Foundation (IRCCS),

Italy

Reviewed by:

Sara L. Weisenbach, Stony Brook Medicine,

United States

Deana Davalos, Colorado State University,

United States

*Correspondence:

Gillberto Sousa Alves

gsalves123@hotmail.com

tThese authors have contributed equally to this work

Specialty section:

This article was submitted to

Aging Psychiatry,

a section of the journal

Frontiers in Psychiatry

Received: 16 September 2019

Accepted: 07 November 2019

Published: 13 December 2019

Citation:

Tavares-Júnior JWL, de Souza ACC,

Alves GS, Bonfadini JdC

Siqueira-Neto Jl and Braga-Neto $P$

(2019) Cognitive Assessment

Tools for Screening Older Adults

With Low Levels of Education:

A Critical Review.

Front. Psychiatry 10:878.

doi: 10.3389/fpsyt.2019.00878

\section{Cognitive Assessment Tools for Screening Older Adults With Low Levels of Education: A Critical Review}

\author{
José Wagner Leonel Tavares-Júnior ${ }^{1 \dagger}$, Ana Célia Caetano de Souza ${ }^{2,3 \dagger}$, \\ Gilberto Sousa Alves ${ }^{*}$, Janine de Carvalho Bonfadini ${ }^{1,3}$, José Ibiapina Siqueira-Neto ${ }^{1}$ \\ and Pedro Braga-Neto ${ }^{1,2,3}$
}

\footnotetext{
${ }^{1}$ Department of Clinical Medicine, Division of Neurology, Universidade Federal do Ceará, Fortaleza, Brazil, ${ }^{2}$ Center of Health Sciences, Universidade Estadual do Ceará, Fortaleza, Brazil, ${ }^{3}$ Neurology Service, Hospital Universitário Walter Cantídio, Universidade Federal do Ceará, Fortaleza, Brazil, ${ }^{4}$ Translational Psychiatry Research Group, Universidade Federal do Maranhão, São Luís, Brazil
}

Introduction: Cognitive assessment of older adults who are either illiterate or with low levels of education is particularly challenging because several battery tasks require a certain educational background. Early detection of mild cognitive impairment (MCl) in the elderly using validated screening tools is of great importance since this population group could benefit from new drugs that are being investigated for the treatment of dementias. Cutoff scores for psychometric properties of cognitive tests are not well established among adults with low levels of education. The present study aimed to critically review the literature on cognitive assessment tools for screening cognitive syndromes including $\mathrm{MCl}$ and Alzheimer's disease (AD) in older adults with low levels of education.

Methods: We conducted a systematic search of MEDLINE, LILACS, Cochrane, and SCOPUS electronic databases of cross-sectional and prospective studies with adults over 55 years of age.

Results: We found a significant number of assessment tools available $(n=44)$, but only a few of them showed diagnostic accuracy for the diagnosis of $\mathrm{MCl}$ and $\mathrm{AD}$ in older adults with low levels of education: the Mini-Mental State Exam; the Montreal Cognitive Assessment; the Persian Test of Elderly for Assessment of Cognition and Executive Function; the Six-Item Screener; and the Memory Alteration Test. Few studies evaluated individuals with low levels of education, with a wide range of cutoff scores and cognitive test batteries.

Conclusion: We found that a small number of studies evaluated adults with 4 years of formal education or less. Our findings further support the importance of developing specific tools for the assessment of older adults with low levels of education.

Keywords: mental status tests, dementia tests, literacy, educational status, mild cognitive impairment 


\section{INTRODUCTION}

Dementia is characterized by cognitive impairment and loss of function (1). The growth of population aging over the past few decades has been associated with an increase in cognitive disorders. Data from Alzheimer's Disease International (ADI) shows there were 46.8 million people living with dementia worldwide in 2015, and it is estimated this number will be 74.5 million in 2030 and 131.5 million in 2050. Alzheimer's disease (AD) is the most common form of dementia and accounts for $50-70 \%$ of dementia cases. There were an estimated 26 million people living with $\mathrm{AD}$ in 2015 and it is believed there will be as many as 41 million by 2030 and 72 million by 2050 (1).

Mild cognitive impairment (MCI) is the intermediate stage between cognitive decline of healthy aging and dementia (2). The prevalence of MCI is $12-18 \%$ among adults over 65 years of age and the annual progression rates from $\mathrm{MCI}$ to $\mathrm{AD}$ are $10-15 \%(3,4)$. Early detection of MCI in the elderly using validated screening tools is of great importance since this population group could benefit from new drugs that are being investigated for the treatment of neurodegenerative diseases including AD. Furthermore, evidence shows that, since MCI is a transitional phase between normal aging and $\mathrm{AD}$, there is less brain involvement and those affected are more likely to benefit from drug therapies $(5,6)$.

Cognitive assessment tools are commonly used for screening impairment, differential diagnosis, determining disease severity, as well as monitoring disease progression in patients (7). A major challenge for the initial assessment of age-related cognitive changes is to find a screening tool that is both sensitive and specific for differential diagnosis of cognitive impairment. Both ceiling effects and floor effects limit the ability of a test or some of its items to accurately assess cognitive decline (8). These effects have been reported in several studies and they are primarily related to educational background (9). The ceiling effect occurs when score distribution is skewed and a measurement is determined by the proportion of people scoring at the high end, thereby preventing to detect health improvements. The opposite is the floor effect that occurs when a measurement is determined by the proportion of people scoring at the low end, thereby preventing to detect health declines (10).

Another important aspect is to have available free, easy-to-use assessment tools that do not require specialized training and have the ability to accurately discriminate cognitive decline in adults with normal aging, MCI, and dementia (4). Cognitive assessment of older adults who are either illiterate or with low levels of education is particularly challenging because several battery tasks require a certain educational background (11-13). There are an estimated 758 million illiterate adults in the world (11) and 13 million people are estimated to be illiterate in Brazil (11). Prospective cohort studies have shown an association between low education and higher risk of developing $\mathrm{AD}$ (14-17). Yet, few studies have examined the performance of cognitive assessment tools in adults with low education.

Cutoff scores for psychometric properties of cognitive tests are not well established among adults with low levels of education. Furthermore, there is a scarcity of studies evaluating assessment tools for screening older adults with low levels of education. A better understanding of the accuracy of different cognitive batteries is crucial for early diagnosis and intervention, and epidemiological studies are needed to further explore how education background affects an individual's performance on different cognitive dimensions.

The present study aimed to critically review the literature on cognitive assessment tools for screening cognitive syndromes including $\mathrm{MCI}$ and $\mathrm{AD}$ in older adults with low levels of education.

\section{METHODS}

An integrative literature review was conducted to gather and summarize the body of evidence available from original articles. This integrative review study included six stages: Step 1-formulation of the central research question (theme identification); Step 2-definition of inclusion and exclusion criteria and literature search; Step 3-categorization of primary studies (definition of data to be extracted from the selected studies); Step 4-assessment of the studies included; Step 5interpretation of results; Step 6-knowledge synthesis of the results obtained from the studies assessed (18-20).

The central research question was formulated using the PVO method where $\mathrm{P}$ is the study population (adults over 55 years of age with low education, i.e., 4 years of formal education or less); $\mathrm{V}$ is the variable (cognitive assessment tools); and $\mathrm{O}$ is the outcome (MCI and $\mathrm{AD}$ ).

The guiding question of our review was: "Which assessment tools are used for cognitive screening of MCI or AD in older adults with low levels of education?" The inclusion criteria were English language articles in the electronic databases Medical Literature Analysis and Retrieval System Online (MEDLINE), Latin American and Caribbean Health Sciences Literature (LILACS), Cochrane, and SCOPUS; cross-sectional or prospective design; outpatient or population-based samples of adults over 55 years of age with low education (4 years of education or less); and assessment of the use of cognitive tools for MCI or AD diagnosis. We did not search the following information sources for this review: guidelines; institutional protocols; self-administered or telephonebased cognitive assessment instruments; and studies that used cognitive assessment instruments for diagnosing other psychiatric or neurological conditions. The publications were individually searched and selected by two investigators during June and July 2019.

The Preferred Reporting Items for Systematic Review and Meta-Analyses (PRISMA) guidelines (21) were used as a basis for the search and selection of studies (Figure 1). A questionnaire was developed to help data extraction (22). Two matrices were constructed to present the results: the first one included study characteristics and the second one included cognitive tools and main results reported.

A search strategy was created to conduct searches in the following databases: MEDLINE via PubMed from the US National Library of Medicine; LILACS; Cochrane; and SCOPUS via Coordenação de Aperfeiçoamento de Pessoal de Nivel Superior 


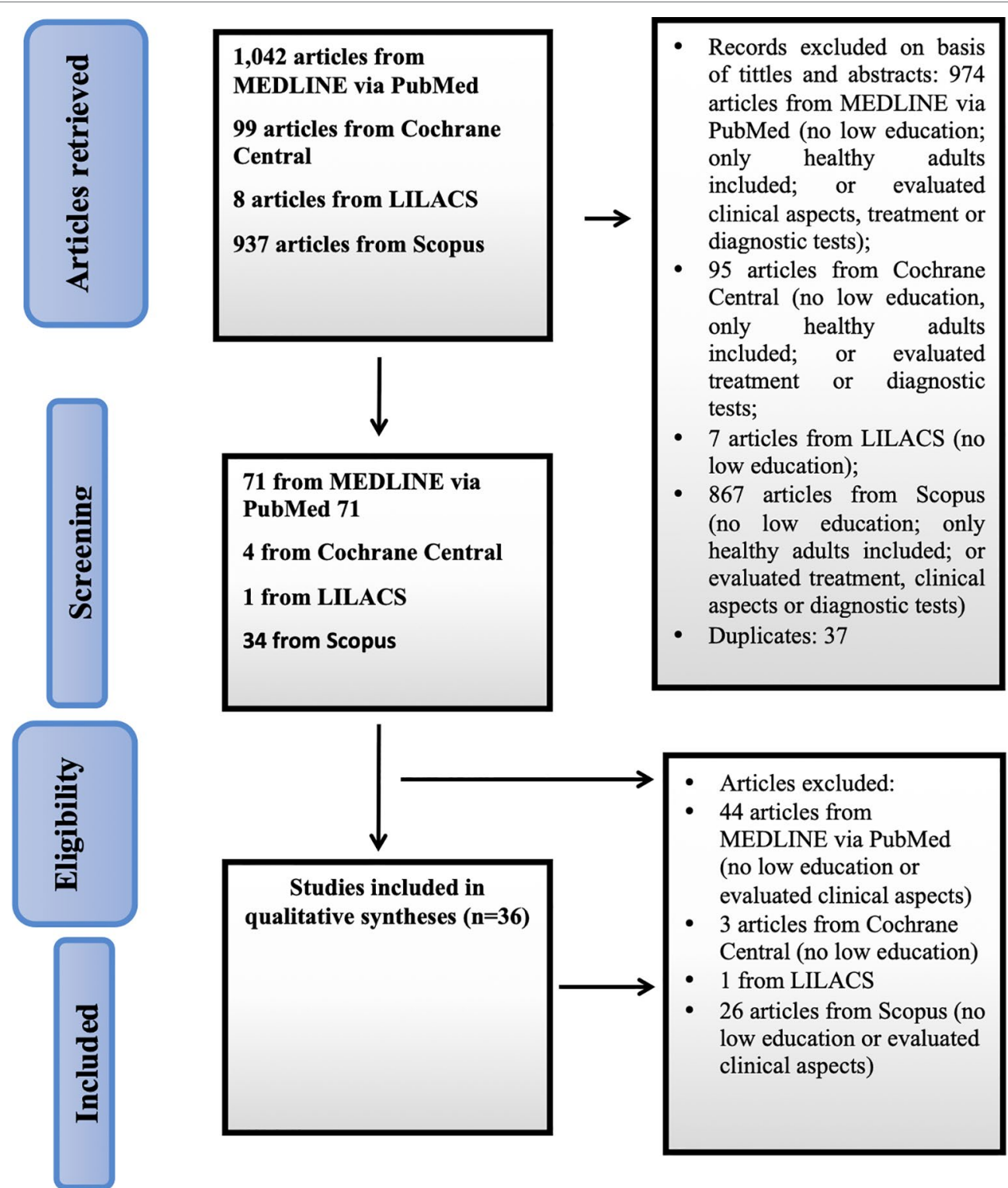

FIGURE 1 | Flowchart of study selection.

(CAPES) with no time restriction. To expand our search, we chose to use natural controlled language. The following descriptors (bold), synonyms, natural language, and Boolean operators were used to cross-check the databases: MEDLINE (Medical Subject Headings [MeSH]: search strategy-(aged or elderly or old or elder) and (literacy or illiteracy or education or "low education") and ("mental status and dementia tests" or tool or instrument or status or test) and ("Alzheimer disease" or alzheimer's) and ("mild cognitive impairment" or "cognitive dysfunction").

To minimize selection bias (misinterpretation of results and study design), the literature search and data extraction were conducted by two investigators independently and any discrepancies were resolved by consensus.

\section{RESULTS}

Figure 1 shows the flowchart of the study selection process according to the PRISMA guidelines. A total of 2,086 articles were retrieved and read. Thirty-six studies were selected for our review.

Table 1 describes the studies assessed. The sample sizes ranged from 50 to 10,432 participants. The studies were conducted in 17 countries, and most of them (13.88\%) were from China and Spain.

A wide range of cognitive assessment tools $(n=44)$ were used for MCI and AD diagnosis (Table 1). Of these, the MiniMental State Examination (MMSE) (59) was the most frequently used $(86.11 \%)$, followed by the Montreal Cognitive Assessment (MoCA) (60) (27.77\%). 


\begin{tabular}{|c|c|c|c|c|c|c|c|c|}
\hline Authors, Year & Country & $\begin{array}{l}\text { Study } \\
\text { Design }\end{array}$ & $\begin{array}{l}\text { Primary Study } \\
\text { Objective }\end{array}$ & Level of Education & Sample Size & Participant Age & $\begin{array}{l}\text { Cognitive } \\
\text { Assessment Tool } \\
\text { used }\end{array}$ & Main Results \\
\hline $\begin{array}{l}\text { Sun Y et al., } \\
2014 \text { (23) }\end{array}$ & Taiwan & $\begin{array}{l}\text { Cross- } \\
\text { sectional }\end{array}$ & $\begin{array}{l}\text { To assess prevalence } \\
\text { of } \mathrm{MCl} \text { and dementia }\end{array}$ & $\begin{array}{l}\text { Illiterate } 32.1 \% \leq 6 \\
\text { years } 45.2 \%>6 \text { years } \\
22.6 \%\end{array}$ & $\begin{array}{l}\text { 10,432 (dementia } \\
\text { 929, MCl 2,049, } \\
\text { indeterminate 419) }\end{array}$ & $\begin{array}{l}\text { Mean age (SD) } 76.2 \\
\text { years (6.7) }\end{array}$ & $\begin{array}{l}\text { MMSE Taiwan } \\
\text { version }\end{array}$ & $\begin{array}{l}\text { Prevalences: dementia } 8.04 \% \\
\text { MCl } 18.76 \%\end{array}$ \\
\hline $\begin{array}{l}\text { Kim KW et al., } \\
2011 \text { (24) }\end{array}$ & South Korea & $\begin{array}{l}\text { Cross- } \\
\text { sectional }\end{array}$ & $\begin{array}{l}\text { To assess prevalence } \\
\text { of } \mathrm{MCl} \text { and dementia } \\
\text { in adults over } 65 \text { years } \\
\text { of age }\end{array}$ & $\begin{array}{l}\text { Illiterate } 31.3 \% 1-6 \\
\text { years } 38.1 \%>7 \text { years } \\
30.6 \%\end{array}$ & 6,141 & $\begin{array}{l}65-69 \text { years } 32.2 \%> \\
70 \text { years } 67.8 \%\end{array}$ & $\begin{array}{l}\text { CERAD-Korean } \\
\text { version, Clinical } \\
\text { Assessment Battery }\end{array}$ & $\begin{array}{l}\text { Prevalences: dementia } 8.1 \% \text {, } \\
\text { AD } 5.7 \%, \mathrm{MCl} 24.1 \%\end{array}$ \\
\hline $\begin{array}{l}\text { Chen MR et al., } \\
2010 \text { (25) }\end{array}$ & China & $\begin{array}{l}\text { Cross- } \\
\text { sectional }\end{array}$ & $\begin{array}{l}\text { To validate SIS for } \\
\text { quick detection of } \\
\text { cognitive impairment }\end{array}$ & $\begin{array}{l}\text { Illiterate } 7.2 \% 1-6 \\
\text { years } 16.4 \%>6 \text { years } \\
76.3 \%\end{array}$ & $\begin{array}{l}\text { 1,976 (healthy aging } \\
475, \mathrm{MCl} 440, \mathrm{AD} \\
1,061 \text { ) }\end{array}$ & $\begin{array}{l}\text { Mean age (SD) } 71.87 \\
\text { years }(8.71)\end{array}$ & SIS, MMSE & $\begin{array}{l}\text { SIS: MCI Sn 34.3\%, Sp } \\
\text { 90.1\%, MMSE: cutoff score } \\
<4 \text { years of schooling } \leq 17 ; \text { Sn } \\
94.3 \% ; \text { Sp } 95.0 \%\end{array}$ \\
\hline $\begin{array}{l}\text { Chang J et al., } \\
2014 \text { (26) }\end{array}$ & Hong Kong & $\begin{array}{l}\text { Cross- } \\
\text { sectional }\end{array}$ & $\begin{array}{l}\text { To assess the effect } \\
\text { of education on tools } \\
\text { for screening older } \\
\text { population }\end{array}$ & $\begin{array}{l}\text { Mean schooling } 4.7 \\
\text { years (SD 4.6; 0-20) }\end{array}$ & $\begin{array}{l}788 \text { (AD 405, } \\
\text { controls 383) }\end{array}$ & $\begin{array}{l}\text { Mean age (SD) } 72.08 \\
\text { years }(7.27)\end{array}$ & $\begin{array}{l}\text { MMSE, ADAS-Cog, } \\
\text { Verbal Fluency, } \\
\text { Abstract Thinking, } \\
\text { and Visual/DS }\end{array}$ & $\begin{array}{l}\text { Effect of educational } \\
\text { background on MMSE, } \\
\text { language sub-item }\end{array}$ \\
\hline $\begin{array}{l}\text { O'Bryant SE et } \\
\text { al., } 2013(27)\end{array}$ & United States & $\begin{array}{l}\text { Cross- } \\
\text { sectional }\end{array}$ & $\begin{array}{l}\text { To characterize a } \\
\text { Mexican American } \\
\text { population with } \mathrm{MCl} \\
\text { and } \mathrm{AD}\end{array}$ & $\begin{array}{l}\text { Mean schooling }(\mathrm{SD}) \\
\text { AD } 5.9 \text { years }(4.5) \\
\text { MCl } 6.6 \text { years }(4.2) \\
\text { Controls } 8.1 \text { years } \\
(4.2)\end{array}$ & $\begin{array}{l}1.069 \text { non-Hispanic } \\
\text { white }(n=633 \text { : AD } \\
\text { 160, MCl 97, controls } \\
\text { 376) Mexican } \\
\text { American ( } n=436, \\
\text { AD 35, MCl 67, } \\
\text { controls 337) }\end{array}$ & $\begin{array}{l}\text { Mean age (SD) AD } 73.6 \\
\text { years }(9.1) \mathrm{MCl} 61.9 \\
\text { years }(12.3) \text { Controls } \\
58.7 \text { years }(9.9)\end{array}$ & MMSE & $\begin{array}{l}\text { Mean MMSE score (SD) AD } \\
\text { 18.5 (5.0) MCl } 24.7 \text { (3.6) } \\
\text { Controls } 27.5(2.8)\end{array}$ \\
\hline $\begin{array}{l}\text { de Paula JJ et } \\
\text { al., } 2013 \text { (28) }\end{array}$ & Brazil & $\begin{array}{l}\text { Cross- } \\
\text { sectional }\end{array}$ & $\begin{array}{l}\text { To validate an } \\
\text { unstructured } \\
\text { neuropsychological } \\
\text { assessment tool for } \\
\text { clinical use }\end{array}$ & $\begin{array}{l}\text { Mean schooling (SD) } \\
\text { MCl } 4.71 \text { years }(4.00) \\
\text { AD } 4.82 \text { years }(3.46) \\
\text { Controls } 5.22 \text { years } \\
(4.29)\end{array}$ & $\begin{array}{l}274 \text { (96 controls, } \mathrm{MCl} \\
85, \mathrm{AD} 93 \text { ) }\end{array}$ & $\begin{array}{l}\text { Mean age }(\mathrm{SD}) \text { Controls } \\
72.61 \text { years }(7.76), \mathrm{MCl} \\
73.18 \text { years }(8.46), \mathrm{AD} \\
74.57 \text { years }(6.65)\end{array}$ & $\begin{array}{l}\text { RAVLT, FAB, verbal } \\
\text { fluency, SDT, CDT, } \\
\text { DS, TT and TN-LIN }\end{array}$ & $\begin{array}{l}\text { Study protocol: } \mathrm{Sn}>70 \%, \mathrm{Sp} \\
>70 \% \text { for } \mathrm{AD} \text { and } \mathrm{MCl}\end{array}$ \\
\hline $\begin{array}{l}\text { Sánchez } \\
\text { Benevides G et } \\
\text { al., } 2014 \text { (29) }\end{array}$ & Spain & $\begin{array}{l}\text { Cross- } \\
\text { sectional }\end{array}$ & $\begin{array}{l}\text { To evaluate } \\
\text { neuropsychological } \\
\text { assessment tool for } \\
\mathrm{MCl} \text { and } \mathrm{AD}\end{array}$ & $\begin{array}{l}\text { Mean schooling (SD) } \\
\text { MCl } 8.0 \text { years }(4.7) \\
\text { AD } 7.6 \text { years }(4.6) \\
\text { Controls } 10.4 \text { years } \\
(5.4)\end{array}$ & $\begin{array}{l}535 \text { (controls } 356 \\
\text { MCl 79, AD 100) }\end{array}$ & $\begin{array}{l}\text { Mean age }(\mathrm{SD}) \text { Controls } \\
64.9 \text { years }(9.3) \mathrm{MCl} \\
72.8 \text { years }(6.5) \mathrm{AD} \\
74.7 \text { years }(7.5)\end{array}$ & $\begin{array}{l}\text { MMSE, DS, WAIS, } \\
\text { TMT, SDMT, BNT, } \\
\text { TT, SVOSPB, JLO, } \\
\text { verbal fluency, } \\
\text { ROCF, FCSRT, } \\
\text { phonemic fluency, } \\
\text { SCWIT, TLDU }\end{array}$ & $\begin{array}{l}\text { Mean MMSE scores (SD) } \\
\text { Controls } 28.7(1.5) \mathrm{MCl} 25.7 \\
\text { (2.2) AD } 20.2(4.0) \mathrm{FCSRT} \\
\text { showed best diagnostic } \\
\text { accuracy for AD vs. controls }\end{array}$ \\
\hline $\begin{array}{l}\text { Mellor D et al., } \\
2016(30)\end{array}$ & China & $\begin{array}{l}\text { Cross- } \\
\text { sectional }\end{array}$ & $\begin{array}{l}\text { To assess } \\
\text { effectiveness for } \\
\text { discriminating } \mathrm{MCl} \\
\text { or } \mathrm{AD} \text { vs. healthy } \\
\text { controls }\end{array}$ & $\begin{array}{l}\text { Mean schooling (SD) } \\
\text { MCI } 5.17(4.78) A D \\
3.72(4.14)\end{array}$ & $\begin{array}{l}1.027 \text { (controls 708, } \\
\text { AD 267, MCl 50) }\end{array}$ & $\begin{array}{l}\text { Mean age }(\mathrm{SD}) 72.54 \\
\text { years }(8.40)\end{array}$ & $\begin{array}{l}\text { MMSE Chinese } \\
\text { version, MoCA }\end{array}$ & $\begin{array}{l}\text { (cutoff score, Sn, Sp) MCl: } \\
\text { MMSE 25.50/68/83 MoCA } \\
\text { 22.50/.87/73 }\end{array}$ \\
\hline $\begin{array}{l}\text { Javadi PSHS et } \\
\text { al., } 2015 \text { (31) }\end{array}$ & Iran & $\begin{array}{l}\text { Cross- } \\
\text { sectional }\end{array}$ & $\begin{array}{l}\text { To characterize } \\
\text { illiterate and literate } \\
\text { older adults; PEACE } \\
\text { scale cutoff scores } \\
\text { for AD }\end{array}$ & $\begin{array}{l}\text { Different levels of } \\
\text { education-illiterate } \\
\text { and literate }\end{array}$ & $\begin{array}{l}101 \text { (controls 33, } \mathrm{MCl} \\
30, \mathrm{AD} 38 \text { ) }\end{array}$ & $\begin{array}{l}\text { Mean age }(\mathrm{SD}) \mathrm{AD} \\
74.60 \text { years }(8.02) \\
\mathrm{MCl} 72.5 \text { years }(7.2) \\
\text { Controls } 67.84 \text { years } \\
(7.29)\end{array}$ & $\begin{array}{l}\text { PEACE, GPCOG, } \\
\text { FAST, MMSE, WMS }\end{array}$ & $\begin{array}{l}\text { MMSE < } 4 \text { years of schooling } \\
\text { MCI } 18.75(1.75) \text { AD } 12.64 \\
(3.78) \text { PEACE AD } 67.5 \text { (Sn } \\
75.8 \%, \text { Sp } 97.4 \%)\end{array}$ \\
\hline
\end{tabular}


TABLE 1 | Continued

\begin{tabular}{|c|c|c|c|c|c|c|c|c|}
\hline Authors, Year & Country & $\begin{array}{l}\text { Study } \\
\text { Design }\end{array}$ & $\begin{array}{l}\text { Primary Study } \\
\text { Objective }\end{array}$ & Level of Education & Sample Size & Participant Age & $\begin{array}{l}\text { Cognitive } \\
\text { Assessment Tool } \\
\text { used }\end{array}$ & Main Results \\
\hline $\begin{array}{l}\text { Chong MS et } \\
\text { al., } 2010 \text { (32) }\end{array}$ & Singapore & $\begin{array}{l}\text { Cross- } \\
\text { sectional }\end{array}$ & $\begin{array}{l}\text { To compare FAB-X } \\
\text { and MMSE for } \\
\text { screening early } \\
\text { cognitive impairment }\end{array}$ & $\begin{array}{l}\text { Mean schooling (SD) } \\
\mathrm{MCl} 7,1 \text { years }(4.4) \\
\text { Controls } 9.6 \text { years } \\
(4.4)\end{array}$ & $\begin{array}{l}180 \text { (controls 100, } \\
\text { MCl 21, dementia 59) }\end{array}$ & $\begin{array}{l}\text { Mean age }(\mathrm{SD}) \text { Controls } \\
63.7 \text { years }(6.51) \mathrm{MCl} \\
69.3 \text { years }(7.91)\end{array}$ & $\begin{array}{l}\text { FAB Chinese } \\
\text { version, MMSE, } \\
\text { verbal fluency, BNT, } \\
\text { WAIS }\end{array}$ & $\begin{array}{l}\text { FAB MCl and mild dementia } \\
\text { Cutoff scores 12/13 (Sn 92\%; } \\
\text { Sp 78.7\%) MMSE (Sn 77\%; } \\
\text { Sp 91.2\%) More effective when } \\
\text { combined }\end{array}$ \\
\hline $\begin{array}{l}\text { Ng A et al., } \\
2013 \text { (33) }\end{array}$ & Singapore & $\begin{array}{l}\text { Cross- } \\
\text { sectional }\end{array}$ & $\begin{array}{l}\text { To assess MoCA } \\
\text { diagnostic accuracy } \\
\text { for } \mathrm{MCl} \text { and } \mathrm{AD} \text { in } \\
\text { older adults }\end{array}$ & $\begin{array}{l}\text { Mean schooling (SD) MCI } \\
10.93 \text { years }(4.28) \mathrm{AD} \\
6.97 \text { years }(4.47) \text { Controls } \\
12.07 \text { years }(3.20)\end{array}$ & $\begin{array}{l}212 \text { (controls 103, } \\
\mathrm{MCl} 49, \mathrm{AD} 60 \text { ) }\end{array}$ & Mean age 62.35 years & MMSE, MoCA & $\begin{array}{l}\text { MoCA cutoff scores } \mathrm{MCl}<26 \\
\text { ( } \leq 10 \text { years of schooling); }<27 \\
(>10 \text { years of schooling), Sn } \\
>94 \%\end{array}$ \\
\hline $\begin{array}{l}\text { Saka E et al., } \\
2006 \text { (34) }\end{array}$ & Turkey & $\begin{array}{l}\text { Cross- } \\
\text { sectional }\end{array}$ & $\begin{array}{l}\text { To assess } \\
\text { performance of } E C R \\
\text { for discriminating } \\
\text { dementia, } A D \text { and } \mathrm{MCl} \\
\text { vs. controls }\end{array}$ & $\begin{array}{l}\text { Mean schooling (SD) } \\
\text { MCl } 8.4 \text { years }(5.0) \\
\text { AD } 6.7 \text { years (4.9) } \\
\text { Controls } 8.4 \text { years } \\
(4.9)\end{array}$ & $\begin{array}{l}113 \text { ( } \mathrm{MCl} 80, \text { controls } \\
33)\end{array}$ & $\begin{array}{l}\text { Mean age (SD) } \mathrm{MCl} \\
69.4 \text { years }(8.3) \mathrm{AD} \\
73.8 \text { years }(6.1) \\
\text { Controls } 72.7 \text { years } \\
(6.7)\end{array}$ & MMSE, ECR & $\begin{array}{l}\text { ECR showed good } \\
\text { performance to discriminate } \\
\text { AD vs. controls (AUC 0.990), } \\
\text { moderate performance for MCl } \\
\text { vs. controls (AUC 0.625) }\end{array}$ \\
\hline $\begin{array}{l}\text { Tognoni G et al., } \\
2005 \text { (35) }\end{array}$ & Italy & $\begin{array}{l}\text { Cross- } \\
\text { sectional }\end{array}$ & $\begin{array}{l}\text { To assess prevalence } \\
\text { of dementia in older } \\
\text { population }\end{array}$ & $\begin{array}{l}<4 \text { years }(15.8 \%),>4 \\
\text { years }(84.2 \%)\end{array}$ & $\begin{array}{l}1.600(\mathrm{AD} 68, \mathrm{MCl} \\
149)\end{array}$ & $\begin{array}{l}\text { Mean age }(S D) 74.65 \\
\text { years }(7.26)\end{array}$ & MMSE, CAMDEX & $\begin{array}{l}\text { Prevalence of amnesic } \mathrm{MCl} \\
4.9 \%, \mathrm{AD} 4.2 \%\end{array}$ \\
\hline $\begin{array}{l}\text { Afgin AE et al., } \\
2012 \text { (36) }\end{array}$ & Israel & $\begin{array}{l}\text { Cross- } \\
\text { sectional }\end{array}$ & $\begin{array}{l}\text { To assess prevalence } \\
\text { of } \mathrm{MCl} \text { and } \mathrm{AD} \text { and } \\
\text { conversion rate from } \\
\mathrm{MCl} \text { to } \mathrm{AD} \text { within a } \\
\text { year or more }\end{array}$ & $\begin{array}{l}0 \text { years } 51 \% 1-4 \\
\text { years } 23 \% 5-8 \text { years } \\
21 \%>8 \text { years } 5 \%\end{array}$ & $\begin{array}{l}944 \text { (controls 497, } \\
\mathrm{MCl} 303, \mathrm{SD} 13, \mathrm{VD} \\
39, \mathrm{AD} 92 \text { ) }\end{array}$ & $\begin{array}{l}\text { Mean age (years) (SD) } \\
\text { DA } 78.5(7.7), \mathrm{MCl} 72.8 \\
(6.1), \text { Controls } 70.7 \\
(5.5) \text { by age groups (\%) } \\
65-69(39.4 \%) 70-79 \\
(46.4 \%) \geq 80(14.1 \%)\end{array}$ & $\begin{array}{l}\text { MMSE Arabic } \\
\text { version, Brookdale } \\
\text { Cognitive Screening } \\
\text { Test }\end{array}$ & $\begin{array}{l}\text { Prevalence AD 9.8\%, MCI } \\
32.1 \% \text { MMSE <4 years of } \\
\text { schooling cutoff scores (SD) } \\
\mathrm{MCl} \text { illiterate } 17.8(1.9), 1-4 \\
\text { years of schooling } 19.6 \text { (3.4); }\end{array}$ \\
\hline $\begin{array}{l}\text { Chaves ML et } \\
\text { al., } 2009 \text { (37) }\end{array}$ & Brazil & Cohort & $\begin{array}{l}\text { To assess incidence } \\
\text { rate of } \mathrm{AD} \text { and } \mathrm{MCl} \text { in } \\
\text { a community of older } \\
\text { adults }\end{array}$ & $\begin{array}{l}\text { Mean schooling }(\mathrm{SD}) \\
9.06 \text { years }(5.50)\end{array}$ & $\begin{array}{l}345 \text { (no details } \\
\text { available) }\end{array}$ & $\begin{array}{l}\text { Mean age }(\mathrm{SD}) 70.37 \\
\text { years }(7.15)\end{array}$ & MMSE & $\begin{array}{l}\text { Incidence rate per 1,000 MCl } \\
\text { 13.2 AD } 14.8\end{array}$ \\
\hline $\begin{array}{l}\text { Custodio N et } \\
\text { al., } 2017 \text { (38) }\end{array}$ & Peru & $\begin{array}{l}\text { Cross- } \\
\text { sectional }\end{array}$ & $\begin{array}{l}\text { MAT performance to } \\
\text { discriminate controls, } \\
\text { MCl and AD in adults } \\
\text { with low education }\end{array}$ & $\begin{array}{l}\text { Mean schooling }(\mathrm{SD}) \\
\mathrm{AD} 2.65 \text { years }(1.28) \\
\mathrm{MCl} 2.53 \text { years }(1.46) \\
\text { Controls } 2.57 \text { years } \\
(1.45)\end{array}$ & $\begin{array}{l}247 \text { (controls 121, } \\
\text { AD 81, } \mathrm{MCl} 45 \text { ) }\end{array}$ & $\begin{array}{l}\text { Mean age }(\mathrm{SD}) \mathrm{AD} \\
74.18 \text { years }(3.81) \mathrm{MCl} \\
71.09 \text { years }(4.20) \\
\text { Controls } 69.53 \text { years } \\
(4.11)\end{array}$ & $\begin{array}{l}\text { MAT, RAVLT, WMS, } \\
\text { TMT A and B, ROCF, } \\
\text { BNT, WCST, DS, } \\
\text { WAIS, MMSE }\end{array}$ & $\begin{array}{l}\text { MAT AD vs. MCI AUC } 99.60 \% \\
\text { MCI vs. controls AUC } 99.56 \% \\
\text { Mean MMSE scores (SD): MCI } \\
\text { 21.36 (0.98); MAT MCI } 30.53 \\
\text { (2.54) }\end{array}$ \\
\hline $\begin{array}{l}\text { Ravaglia G et } \\
\text { al., } 2008 \text { (39) }\end{array}$ & Italy & Cohort & $\begin{array}{l}\text { To assess incidence } \\
\text { and prevalence rates } \\
\text { of } \mathrm{MCl} \text { in older adults }\end{array}$ & $\begin{array}{l}\text { Mean schooling (SD) } \\
4.3 \text { years }(2.3)\end{array}$ & $\begin{array}{l}1.016 \text { (controls } 865 \text {, } \\
\text { MCl } 75 \text {, dementia } 60, \\
\text { indeterminate } 19 \text { ) }\end{array}$ & $\begin{array}{l}\text { Mean age }(\mathrm{SD}) \text { Controls } \\
73.6 \text { years }(6.1) \mathrm{MCl} \\
78.1 \text { years }(8.3)\end{array}$ & $\begin{array}{l}\text { MMSE, Mental } \\
\text { Deterioration Battery }\end{array}$ & $\begin{array}{l}\mathrm{MCl} \text { prevalence } 7.7 \% \text {; } \\
\text { incidence rate in } 4 \text { years } 76.8 \\
\text { per 1,000 persons-years }\end{array}$ \\
\hline $\begin{array}{l}\text { Rahman TTA et } \\
\text { al., } 2009 \text { (40) }\end{array}$ & Egypt & $\begin{array}{l}\text { Cross- } \\
\text { sectional }\end{array}$ & $\begin{array}{l}\text { To assess validity } \\
\text { of } \mathrm{MoCA} \text { version to } \\
\text { detect } \mathrm{MCl}\end{array}$ & $\begin{array}{l}\text { Mean schooling } \mathrm{MCl} \\
8.2 \text { years (5.5) }\end{array}$ & $\begin{array}{l}184 \text { (MCl 94, controls } \\
90)\end{array}$ & $\begin{array}{l}\text { Mean age (SD) } 64.5 \\
\text { years }(6.8)\end{array}$ & $\begin{array}{l}\text { MoCA Arabic } \\
\text { version, CAMCOG }\end{array}$ & $\begin{array}{l}\text { MoCA MCI Sn 92.3\% Sp } \\
85.7 \%\end{array}$ \\
\hline $\begin{array}{l}\text { Freitas S et al., } \\
2013(41)\end{array}$ & Portugal & $\begin{array}{l}\text { Cross- } \\
\text { sectional }\end{array}$ & $\begin{array}{l}\text { To validate MoCA } \\
\text { for screening of } \mathrm{MCl} \\
\text { and } \mathrm{AD}\end{array}$ & $\begin{array}{l}\text { Mean schooling (SD) } \\
\text { MCI } 6.50 \text { years }(4.56) \\
\text { AD } 6.2 \text { years }(4.11) \\
\text { Controls } 6.39 \text { years } \\
(4.30)\end{array}$ & $\begin{array}{l}360 \text { (MCl 90, AD 90, } \\
\text { controls 180) }\end{array}$ & $\begin{array}{l}\text { Mean age }(\mathrm{SD}) 71.86 \\
\text { years }(7.895)\end{array}$ & MMSE, MoCA & $\begin{array}{l}\text { Cutoff scores/Sn/Sp MCl } \\
\text { MoCA <22/81/77, MMSE } \\
<29 / 67 / 72\end{array}$ \\
\hline
\end{tabular}


TABLE 1 | Continued

\begin{tabular}{|c|c|c|c|c|c|c|c|c|}
\hline Authors, Year & Country & $\begin{array}{l}\text { Study } \\
\text { Design }\end{array}$ & $\begin{array}{l}\text { Primary Study } \\
\text { Objective }\end{array}$ & Level of Education & Sample Size & Participant Age & $\begin{array}{l}\text { Cognitive } \\
\text { Assessment Tool } \\
\text { used }\end{array}$ & Main Results \\
\hline $\begin{array}{l}\text { Borson S et al., } \\
2005 \text { (42) }\end{array}$ & United States & $\begin{array}{l}\text { Cross- } \\
\text { sectional }\end{array}$ & $\begin{array}{l}\text { To compare the Mini- } \\
\text { Cog Test vs. MMSE } \\
\text { for screening cognitive } \\
\text { impairment }\end{array}$ & $\begin{array}{l}\text { Literate } 76 \% \text {, Semi- } \\
\text { literate and illiterate } \\
24 \%\end{array}$ & $\begin{array}{l}371 \text { (controls 140, } \\
\text { AD 112, MCl 71, } \\
\text { other dementias 48) }\end{array}$ & $\begin{array}{l}\text { Mean age Controls } 73 \\
\text { years } \mathrm{MCl} 74 \text { years } A D \\
78 \text { years }\end{array}$ & $\begin{array}{l}\text { CASI, MMSE, } \\
\text { Mini-Cog }\end{array}$ & $\begin{array}{l}\mathrm{AD} \text { and } \mathrm{MCl} \text { diagnostic } \\
\text { accuracy Mini-Cog } 83 \% \\
\text { MMSE } 81 \%\end{array}$ \\
\hline $\begin{array}{l}\text { Boycheva E et } \\
\text { al., } 2018 \text { (43) }\end{array}$ & Spain & $\begin{array}{l}\text { Cross- } \\
\text { sectional }\end{array}$ & $\begin{array}{l}\text { To assess clinical } \\
\text { performance of MDRS } \\
\text { for screening } \mathrm{MCl} \text { and } \\
\mathrm{AD} \text { in older adults }\end{array}$ & $\begin{array}{l}\text { Mean schooling (SD) } \\
7.08 \text { years (3.57) }\end{array}$ & $\begin{array}{l}125 \text { (AD 45, MCl } 37 \\
\text { controls 43) }\end{array}$ & $\begin{array}{l}\text { Mean age } 75.12 \text { years } \\
(6.83)\end{array}$ & $\begin{array}{l}\text { MDRS, MMSE, } \\
\text { WAT, FCSRT, BNT, } \\
\text { SCWIT, GSBT, verbal } \\
\text { fluency, phonemic } \\
\text { fluency, WAIS }\end{array}$ & $\begin{array}{l}\text { MDRS-2 cutoff score MCl } \\
\text { vs. controls } 131 \text { (Sn 89\%, Sp } \\
81 \%)\end{array}$ \\
\hline $\begin{array}{l}\text { Pezzotti P et al., } \\
2008 \text { (44) }\end{array}$ & Italy & $\begin{array}{l}\text { Cross- } \\
\text { sectional }\end{array}$ & $\begin{array}{l}\text { To compare } \\
\text { agreement of MMSE } \\
\text { between primary } \\
\text { care and specialist } \\
\text { practitioners }\end{array}$ & $\begin{array}{l}0-5 \text { years } 68.1 \%>5 \\
\text { years } 31.9 \%\end{array}$ & $\begin{array}{l}317(\mathrm{MCl} 40, \mathrm{AD} 95, \\
\text { other dementias } 98, \\
\text { healthy controls } 84)\end{array}$ & Mean age not available & $\begin{array}{l}\text { MMSE, Mental } \\
\text { Deterioration Battery, } \\
\text { SCWIT }\end{array}$ & $\begin{array}{l}\text { Mean MMSE score Primary } \\
\text { care providers } 15.8 \text { Specialists } \\
17.4 \text { (kappa } 0.86 \text { ) }\end{array}$ \\
\hline $\begin{array}{l}\text { Julayanont P et } \\
\text { al., } 2015(45)\end{array}$ & Thailand & $\begin{array}{l}\text { Cross- } \\
\text { sectional }\end{array}$ & $\begin{array}{l}\text { To assess validity of } \\
\mathrm{MoCA}-\mathrm{B} \text { for } \mathrm{MCl} \text { in a } \\
\text { population with low } \\
\text { education }\end{array}$ & $\begin{array}{l}\text { Mean schooling (SD) } \\
\mathrm{MCl} 2.9 \text { years (1.7) }\end{array}$ & $\begin{array}{l}85 \text { (controls } 43, \mathrm{MCl} \\
42 \text { ) }\end{array}$ & $\begin{array}{l}\text { Mean age (SD) } \mathrm{MCl} \\
70.2 \text { years }(6.6)\end{array}$ & $\begin{array}{l}\text { MoCA-B, MMSE } \\
\text { Thai version }\end{array}$ & $\begin{array}{l}\text { Mean score (SD) MMSE MCI } \\
18.9(3.0) \text { MoCA-B } 21.3(3.8)\end{array}$ \\
\hline $\begin{array}{l}\text { Matías-Guiu JA } \\
\text { et al., } 2017 \text { (46) }\end{array}$ & Spain & $\begin{array}{l}\text { Cross- } \\
\text { sectional }\end{array}$ & $\begin{array}{l}\text { To validate LASSI-L } \\
\text { scale for } \mathrm{MCl} \text { and } \mathrm{AD} \\
\text { diagnosis }\end{array}$ & $\begin{array}{l}\text { Mean schooling (SD) } \\
\text { Controls } 8.52 \text { years } \\
(4.98), \text { MCI } 7.61 \text { years } \\
(4.79) \text { AD } 7.06 \text { years } \\
(4.20)\end{array}$ & $\begin{array}{l}164 \text { (controls } 97, \mathrm{MCl} \\
34, \mathrm{AD} 33 \text { ) }\end{array}$ & $\begin{array}{l}\text { Mean age (SD) } 73.4 \\
\text { years (10.0) }\end{array}$ & LASSI-L, MMSE & $\begin{array}{l}\text { LASSI-L discrimination AD vs. } \\
\text { controls AUC 0.986; MCI vs. } \\
\text { controls AUC 0.909 }\end{array}$ \\
\hline $\begin{array}{l}\text { Chu LW et al., } \\
2015 \text { (47) }\end{array}$ & Hong Kong & $\begin{array}{l}\text { Cross- } \\
\text { sectional }\end{array}$ & $\begin{array}{l}\text { To validate MoCA } \\
\text { Chinese version for } \\
\text { screening } \mathrm{MCl} \text { and } \mathrm{AD} \\
\text { in older adults }\end{array}$ & $\begin{array}{l}\text { Mean schooling (SD) } \\
\text { Controls } 6.97 \text { years } \\
(4.69) \mathrm{MCl} 4.62 \text { years } \\
(5.19) \mathrm{AD} 4.56 \text { years } \\
(5.00)\end{array}$ & $\begin{array}{l}266 \text { (controls 115, } \\
\mathrm{MCl} 87, \mathrm{AD} 64 \text { ) }\end{array}$ & Mean age 75.3 years & $\begin{array}{l}\text { MoCA Cantonese } \\
\text { Chinese version, } \\
\text { MMSE Chinese } \\
\text { version }\end{array}$ & $\begin{array}{l}\text { MoCA cutoff score/Sn/Sp } \\
\text { MCl 22-3/78\%/73\%, AD } \\
\text { 19-20/94\%/92\% }\end{array}$ \\
\hline $\begin{array}{l}\text { Kurt P et al., } \\
2014(48)\end{array}$ & Turkey & $\begin{array}{l}\text { Cross- } \\
\text { sectional }\end{array}$ & $\begin{array}{l}\text { To develop a } \\
\text { composite score for } \\
\text { DEKOD in an older } \\
\text { population }\end{array}$ & $\begin{array}{l}<4 \text { years of schooling } \\
45.7 \%>5 \text { years of } \\
\text { schooling } 54.3 \%\end{array}$ & $\begin{array}{l}444 \text { (controls 338, } \\
\text { dementia 53, MCl 53) }\end{array}$ & $\begin{array}{l}\text { Mean age (SD) controls } \\
70.7 \text { years }(5.4) \\
\text { dementia } 74 \text { years }(7.8) \\
\mathrm{MCl} 71.7 \text { years }(5.6)\end{array}$ & $\begin{array}{l}\text { DEKOD, MMSE } \\
\text { Turkish version }\end{array}$ & $\begin{array}{l}\text { MMSE }<4 \text { years of schooling: } \\
\text { cutoff score/Sn/Sp Dementia } \\
\text { 17-18/95\%/83\% MCl } \\
22-23 / 67 \% / 55 \% \text { DEKOD } \\
\text { Dementia } 49-50 / 91 \% / 90 \% \\
\text { MCl 60-61/70\%/65\% }\end{array}$ \\
\hline $\begin{array}{l}\text { Bae JB et al., } \\
2015 \text { (49) }\end{array}$ & South Korea & $\begin{array}{l}\text { Cross- } \\
\text { sectional }\end{array}$ & $\begin{array}{l}\text { To assess incidence } \\
\text { rates of } \mathrm{AD} \text { and } \mathrm{MCl} \\
\text { within } 3.5 \text { years of } \\
\text { follow-up }\end{array}$ & $\begin{array}{l}\text { Mean schooling }(S D) \\
6.8 \text { years }(5.4)\end{array}$ & 181 (not available) & Mean age 71.7 years & $\begin{array}{l}\text { MMSE, Korean } \\
\text { CERAD, Clinical } \\
\text { Assessment Battery, } \\
\text { CERAD-K-N }\end{array}$ & $\begin{array}{l}\text { Incidence rate per } 1,000 \\
\text { persons-years } A D 7.9 \mathrm{MCl} \\
28.1\end{array}$ \\
\hline $\begin{array}{l}\text { Paddick SM et } \\
\text { al., } 2015 \text { (50) }\end{array}$ & Tanzania & Cohort & $\begin{array}{l}\text { To assess outcomes, } \\
\text { prevalence and } \\
\text { profiles of patients } \\
\text { with } \mathrm{MCl} \text { in a rural } \\
\text { community }\end{array}$ & $\begin{array}{l}\text { Illiterate with } \mathrm{MCl} \\
55.5 \% \text { Literate (at } \\
\text { least elementary } \\
\text { schooling) } 44.5 \%\end{array}$ & $\begin{array}{l}296(\mathrm{MCl} 46, \\
\text { dementia 78, controls } \\
\text { 172) }\end{array}$ & Mean age 82 years & CERAD & Prevalence of $\mathrm{MCl} 7 \%$ \\
\hline $\begin{array}{l}\text { Choi SJ et al., } \\
2008 \text { (51) }\end{array}$ & South Korea & $\begin{array}{l}\text { Cross- } \\
\text { sectional }\end{array}$ & $\begin{array}{l}\text { To assess prevalence } \\
\text { of } \mathrm{AD} \text { and } \mathrm{MCl} \text { in older } \\
\text { Korean adults }\end{array}$ & $\begin{array}{l}\text { Mean schooling }(\mathrm{SD}) \\
\text { MCl } 4.93 \text { years }(3.27) \\
\text { DA } 1.69 \text { years }(3.09)\end{array}$ & $\begin{array}{l}175 \text { (controls 102, } \\
\mathrm{MCl} 57, \mathrm{AD} 16)\end{array}$ & $\begin{array}{l}\text { Mean age (SD) } 74.3 \\
\text { years (16.7) }\end{array}$ & $\begin{array}{l}\text { Korean MMSE, } \\
\text { CERAD-K }\end{array}$ & $\begin{array}{l}\text { Prevalences: AD 9.0\% MCl } \\
32.9 \%\end{array}$ \\
\hline
\end{tabular}




\begin{tabular}{|c|c|c|c|c|c|c|c|c|}
\hline Authors, Year & Country & $\begin{array}{l}\text { Study } \\
\text { Design }\end{array}$ & $\begin{array}{l}\text { Primary Study } \\
\text { Objective }\end{array}$ & Level of Education & Sample Size & Participant Age & $\begin{array}{l}\text { Cognitive } \\
\text { Assessment Tool } \\
\text { used }\end{array}$ & Main Results \\
\hline $\begin{array}{l}\text { Gavrila D et al., } \\
2009 \text { (52) }\end{array}$ & Spain & $\begin{array}{l}\text { Cross- } \\
\text { sectional }\end{array}$ & $\begin{array}{l}\text { To assess prevalence } \\
\text { of } \mathrm{MCl} \text { and dementia } \\
\text { in older adults }\end{array}$ & $\begin{array}{l}\text { Illiterate } 7.8 \% \text { Less } \\
\text { than elementary } \\
19.7 \% \text {, Elementary or } \\
\text { more } 72.5 \%\end{array}$ & $\begin{array}{l}\text { 1,017 (controls } 726 \\
\text { MCl 235, AD 30, } \\
\text { other dementias 26) }\end{array}$ & $\begin{array}{l}\text { Mean age (SD) } 73.9 \\
\text { years (6.8) }\end{array}$ & $\begin{array}{l}\text { MMSE, CAMDEX, } \\
\text { Blessed Dementia } \\
\text { Scale }\end{array}$ & $\begin{array}{l}\text { Prevalence of amnesic } \mathrm{MCl} \\
8.7 \%\end{array}$ \\
\hline $\begin{array}{l}\text { Wang Bet al., } \\
2011 \text { (53) }\end{array}$ & China & $\begin{array}{l}\text { Cross- } \\
\text { sectional }\end{array}$ & $\begin{array}{l}\text { To describe clinical } \\
\text { characteristics of } \\
\text { patients in a memory } \\
\text { center }\end{array}$ & $\begin{array}{l}0 \text { years } 8 \% 1-5 \text { years } \\
15 \%>6 \text { years } 77 \%\end{array}$ & $\begin{array}{l}\text { 2,789 (healthy aging } \\
604, \mathrm{MCl} 635 \\
\text { AD 1084, other } \\
\text { diagnoses 466) }\end{array}$ & $\begin{array}{l}<50 \text { years } 5.2 \% 50-59 \\
\text { years } 14.9 \%>60 \\
\text { years } 79.9 \%\end{array}$ & MMSE & $\begin{array}{l}\text { Population distribution in a } \\
\text { memory center: } \mathrm{AD} 83.7 \% \\
\mathrm{MCl} 22.8 \%\end{array}$ \\
\hline $\begin{array}{l}\text { Zhou Y et al., } \\
2015 \text { (54) }\end{array}$ & United States & $\begin{array}{l}\text { Cross- } \\
\text { sectional }\end{array}$ & $\begin{array}{l}\text { How to adjust MoCA } \\
\text { for educational } \\
\text { background in a } \\
\text { Spanish-speaking } \\
\text { population }\end{array}$ & $\begin{array}{l}\text { Mean schooling (SD) } \\
\text { Controls } 10.3 \text { years } \\
\text { (6.4) MCl } 7.1 \text { years } \\
\text { (4.8) Dementia } 6.8 \\
\text { years (5.5) }\end{array}$ & $\begin{array}{l}50(\mathrm{AD} 18, \mathrm{MCl} 21, \\
\text { controls } 6, \text { other } \\
\text { dementias } 5)\end{array}$ & $\begin{array}{l}\text { Mean age (SD) } 71.4 \\
\text { years }(9.7)\end{array}$ & MoCA & $\begin{array}{l}\text { It was required to adjust } \\
\text { scores } 3-4 \text { points for those }<6 \\
\text { years of schooling }\end{array}$ \\
\hline $\begin{array}{l}\text { Chen } \mathrm{K} \text { et al., } \\
2016 \text { (55) }\end{array}$ & China & $\begin{array}{l}\text { Cross- } \\
\text { sectional }\end{array}$ & $\begin{array}{l}\text { To assess } \\
\text { performance of } \\
\text { MoCA-B Chinese } \\
\text { version for screening } \\
\mathrm{MCl} \text { in older adults }\end{array}$ & $\begin{array}{l}\text { Groups by years of } \\
\text { schooling }(<6,7-12 \text {, } \\
>12) \text {. Mean schooling } \\
\text { (SD) of those with }<6 \\
\text { years: controls } 4.8 \\
\text { years (1.7), MCl } 3.3 \\
\text { years (2.4), AD } 3.7 \\
\text { years (2.5) }\end{array}$ & $\begin{array}{l}704 \text { (MCl 264, AD } \\
160, \text { controls 280) }\end{array}$ & $\begin{array}{l}\text { Mean age (SD) of } \\
\text { those with }<6 \text { years of } \\
\text { schooling controls } 68.2 \\
\text { years }(9.1) \mathrm{MCl} 68.5 \\
\text { years (8.5) AD } 67.9 \\
\text { years (9.4) }\end{array}$ & $\begin{array}{l}\text { MMSE, AVLT, ROCF, } \\
\text { BNT, Verbal Fluency, } \\
\text { TMT, SCWIT, SDMT, } \\
\text { MoCA-B Chinese } \\
\text { version }\end{array}$ & $\begin{array}{l}\text { Cutoff scores Sn/Sp of those } \\
\text { with }<6 \text { years of schooling: } \\
\text { MCI MoCA-BC 19/87.9/81.0 } \\
\text { MMSE 26/86.2/60.3 }\end{array}$ \\
\hline $\begin{array}{l}\text { Matías-Guiu JA } \\
\text { et al., } 2017 \text { (56) }\end{array}$ & Spain & $\begin{array}{l}\text { Cross- } \\
\text { sectional }\end{array}$ & $\begin{array}{l}\text { To compare diagnostic } \\
\text { properties of five } \\
\text { cognitive screening } \\
\text { tools }\end{array}$ & $\begin{array}{l}\text { Mean schooling (SD) } \\
\text { Controls } 8.01 \text { years } \\
(5.40) \text { AD } 7.10 \text { years } \\
(4.07)\end{array}$ & $\begin{array}{l}160 \text { (AD 92, controls } \\
68 \text { ) }\end{array}$ & $\begin{array}{l}60-69 \text { years } 7.5 \% \\
70-79 \text { years } 48.8 \% \\
80-93 \text { years } 43.8 \%\end{array}$ & $\begin{array}{l}\text { MMSE, ACE-III, MIS, } \\
\text { MoCA, RUDAS }\end{array}$ & $\begin{array}{l}\text { AD: ACE-III AUC 0.897; } \\
\text { RUDAS AUC 0.889; MMSE } \\
\text { AUC 0.874; MIS AUC 0.866; } \\
\text { MoCA AUC } 0.856\end{array}$ \\
\hline $\begin{array}{l}\text { Huang } Y Y \text { et al., } \\
2019(57)\end{array}$ & China & $\begin{array}{l}\text { Cross- } \\
\text { sectional }\end{array}$ & $\begin{array}{l}\text { To compare diagnostic } \\
\text { accuracy of MoCA-B } \\
\text { and MoCA-Beijing } \\
\text { for screening MCl in } \\
\text { people with different } \\
\text { levels of education }\end{array}$ & $\begin{array}{l}\text { No supplementary } \\
\text { table available }\end{array}$ & $\begin{array}{l}808 \text { ( } \mathrm{MCl} 295, \mathrm{AD} \\
254 \text {, controls 259) }\end{array}$ & Not available & $\begin{array}{l}\text { MoCA, MMSE, } \\
\text { AVLT; ROCF, BNT, } \\
\text { Verbal Fluency, TMT, } \\
\text { SCWIT, SDMT }\end{array}$ & $\begin{array}{l}\text { Performance in discriminating } \\
\mathrm{MCl} \text { vs. controls: MoCA B } \\
\text { AUC } 0.95 \text { MoCA-BJ AUC } 0.87\end{array}$ \\
\hline $\begin{array}{l}\text { Khedr E et al., } \\
2015 \text { (58) }\end{array}$ & Egypt & $\begin{array}{l}\text { Cross- } \\
\text { sectional }\end{array}$ & $\begin{array}{l}\text { To assess prevalence } \\
\text { of } \mathrm{MCl} \text { and dementia } \\
\text { in Egyptian adults over } \\
60 \text { years of age }\end{array}$ & $\begin{array}{l}\mathrm{MCl} 75 \% \text { illiterate AD } \\
75 \% \text { illiterate }\end{array}$ & $\begin{array}{l}691 \text { ( } \mathrm{MCl} 12 \\
\text { dementia 35) }\end{array}$ & $\begin{array}{l}\text { Mean age }(\mathrm{SD}) \mathrm{MCl} \\
67.3 \text { years }(7.1), \mathrm{AD} \\
69.3 \text { years }(7.7)\end{array}$ & ${ }^{*} \mathrm{MMSE}, \mathrm{WMS}$ & $\mathrm{MCl}$ prevalence 1.72/100 \\
\hline
\end{tabular}

MMSE, Mini-Mental State Examination; BNT, Boston Naming Test; AVLT, Auditory Verbal Learning Test; MoCA, Montreal Cognitive Assessment; MoCA B, MoCA Basic; ACE, Addenbrooke Cognitive Examination; TMSE, Taiwanese Mental State Examination; CERAD, Consortium to Establish a Registry for Alzheimer's Disease Assessment Packet; SDT, Stick Design Test; TT, Token Test; SDMT, Symbol Digit Modalities Test; SVOSPB, Selected test of the Visual Object and Space Perception Battery; JLO, Judgment of Line Orientation; TLDU, Tower of London Drexel University version; WAT, Word Accentuation Test; GSBT, Gesture Sequences subtest from the Barcelona Test; SIS, Six-Item Screener; ADAS-Cog, Alzheimer's Disease Assessment Scale's cognitive subscale; RAVLT, Rey-Auditory Verbal Learning Test; FAB, Frontal Assessment Battery; CDT, Clock Drawing Test; DS, Digit Span; TMT, Trail Making Test; ROCF, Rey-Osterrieth Complex Figure; FCSRT, Free and Cued Selective Reminding Test; SCWIT, Stroop Color-Word Interference Test; PEACE, Persian Test of Elderly for Assessment of Cognition and Executive function; GPCOG, General Practitioner assessment of Cognition; FAST, Functional Assessment Staging; WMS, Wechsler Memory scale; ECR, Enhanced cued recall test; CAMDEX, Cambridge Examination for Mental Disorders of the Elderly; WAIS, Wechsler Adult Intelligence Scale-Revised; MDRS, Mattis Dementia Rating Scale; BDAE, Boston Diagnostic Aphasia Examination; RDT, Rosen Drawing Test; MAT, Memory Alteration Test; WCST, Wisconsin Card Sorting Test; CASI, Cognitive Abilities Screening Instrument; LASSI-L, Loewenstein-Acevedo Scale for Semantic Interference and Learning; DEKOD, Dokuz Eylül Kognitif Degerlendirme/Dokuz Eylul Cognitive Assessment; CERAD-K-N, CERAD-K Neuropsychological Assessment Battery; MIS, Memory Impairment Screen; AUC, Area Under the ROC Curve; CAMCOG, Cambridge Cognitive Examination; RUDAS, Rowland Universal Dementia Assessment Scale. 
The studies included in our review evaluated adults with different educational backgrounds. However, detailed information was not available in all studies. Adults with $\mathrm{AD}$ had 1.69 (51) to 7.6 years (29) of schooling and those with MCI had 2.53 (38) to 10.93 years (33) of schooling. The proportion of illiterate adults ranged from $1.8 \%$ (35) to $32.1 \%$ (23).

Most were cross-sectional studies (91.67\%), followed by cohort studies (8.33\%). Some of the studies (23, 24, 35, 36, 39, 50-52, 58) assessed the prevalence of MCI and AD ranging from $1.72 \%(58)$ to $32.9 \%(52)$ and $4.2 \%(35)$ to $9 \%(51)$, respectively. The number of adults with MCI ranged from 12 (58) to 2,049 (30) and the number of adults with AD ranged from 16 (51) to 1,061 (25). Mean age of the study participants ranged from 64.5 (40) to 82 years (50).

The Persian Test of Elderly for Assessment of Cognition and Executive Function (PEACE) was used in one study (28) to establish cutoff scores in individuals with AD. The sample consisted of 38 subjects with $\mathrm{AD}$; some of them were illiterate (proportion not available). A cutoff score of 67.5 was set (sensitivity $=75.8 \%$; specificity $=97.4 \%$ ). The Six-Item Screener (SIS) was used in another study (25) that evaluated 440 individuals with MCI with a small proportion of individuals $(<25 \%)$ with low education $(<$ 6 years of schooling). The SIS showed low sensitivity for screening MCI in this population (sensitivity $=34.3 \%$; specificity $=90.1 \%$ ). The Memory Alteration Test (MAT) was used in a single study (38) for discriminating MCI and AD from healthy individuals. The AUC of MAT to discriminate between early AD and amnestic mild cognitive impairment (aMCI) was 99.60\% (sensitivity $=100.00 \%$; specificity $=97.53 \%)$ and to discriminate between aMCI and controls was $99.56 \%$ (sensitivity $=99.17 \%$; specificity $=91.11 \%$ ). The mean score was $17.54 \pm 4.67$ for individuals with $\mathrm{AD}, 30.53 \pm 2.54$ for individuals with $\mathrm{MCI}$ and $41.97 \pm 2.6$ for healthy individuals. $\mathrm{AD}$ and MCI individuals and controls had on average $2.65 \pm 1.28$, $2.53 \pm 1.46$, and $2.57 \pm 1.45$ years of education, respectively.

In this review, six studies (Table 2) assessed cutoff scores of the MMSE for adults with 4 years of education or less $(25,36,38,40,45$, 48). MCI cutoff scores (SD) ranged from 17.8 (1.9) to 21.36 (0.98), but there was great variation in sensitivity and specificity. The Montreal Cognitive Assessment-Basic (MoCA-B) was evaluated in three studies $(45,55,57)$. One of these studies established a cutoff score of 19 for detecting MCI, with $87.9 \%$ sensitivity and $81.0 \%$ specificity (55). Another one reported a cutoff score (SD) of 21.3 (3.8) for detecting MCI in adults with 4 years of education or less (45). Cutoff scores (SD) for AD ranged from 12.64 (3.78) to 18.32 (2.78) in these same studies. Another cognitive test battery reported was the Addenbrooke's Cognitive Examination Revised (ACE$R$ ), which was used in only one study but the cutoff score was not adjusted for low educational level. Mean ACE-R scores were 78.12 (12.79) for controls and 53.20 (14.76) for AD. This tool showed good diagnostic accuracy for diagnosing AD (AUC = 0.897) (25).

\section{DISCUSSION}

We carried out a critical review of cognitive assessment tools for screening cognitive syndromes in older adults with low levels of education. A significant number of assessment tools $(n=44)$ were used in the studies reviewed, but only a few of them showed diagnostic accuracy for the diagnosis of MCI and AD in adults with low education including MMSE, MoCA, PEACE, SIS, and MAT. The latter three were each used in one study only.

It is crucial to validate cognitive assessment instruments in populations with low education and to establish cutoff scores for screening these individuals in daily clinical practice. It would enable to monitoring healthy aging in such a quite large population (11) and evaluate older adults with low levels of education who are at risk of developing dementia syndromes (61). Besides, it could offer new insights to better understand the influence of education on cognitive reserve since there is a relationship between literacy and the functional organization of the human brain. Literacy acquisition improves early visual processing and phonological information processing (62). Indeed, functional neuroimaging studies have evidenced that large neural networks in both cerebral hemispheres have less functional connections in less educated individuals (63). Since there has been a move toward the development of diseasemodifying drugs for $\mathrm{AD}$, it will be paramount to have validated diagnostic tools for population-based assessments including older adults with low levels of education (64).

In agreement with literature reports, the MMSE was the most frequently used cognitive tool in the studies assessed (65). The MMSE is easy to administer and requires no specialized training and it has been validated in many countries (66). We assessed in our review a study that showed good agreement of the MMSE for cognitive screening $(\kappa=0.86)$ between primary care and specialist practitioners (44). However, studies have demonstrated the effect of education on MMSE scores. The MMSE has low sensitivity for MCI, does not perform well in assessing executive functions and has limiting floor and ceiling effects $(9,23,40)$. A study conducted in Brazil has established MMSE cutoff scores of 20 for illiterate adults and 25 for those with 4 years of education or less (67).

The second most frequently used cognitive tool was the MoCA. The MoCA is a cognitive battery that includes tests sensitive to executive functions and has higher sensitivity for diagnosing MCI $(24,33,68)$. However, MoCA scores are strongly influenced by educational background as MoCA tasks are designed for a certain level of education making it difficult to assess individuals who are either illiterate or with low levels of education (30). Therefore, a MoCA basic version (MoCA-B) was developed to include tasks designed to assess the same cognitive domain regardless of the level of education $(45,60,68)$.

A systematic review of cognitive screening tools showed that the ACE- $\mathrm{R}$ is an outstanding test battery. It takes approximately 20 minutes to be administered and it includes tasks designed for different levels of schooling $(52,58)$. In our review, ACE-R cutoff scores for low education were not available.

The studies assessed included recommendations of specific cutoff scores and scales for diagnosis of MCI and AD in adults with low levels of education. One study using the MoCA-B suggested a cutoff score of 19 (55) for MCI diagnosis. For AD, one study showed a cutoff score of 23.5 (30) for the MMSE and another study suggested a cutoff score of 17 (41) for the MoCA.

The scarcity of cognitive assessment tests for evaluating adults with low levels of education is in line with the challenge 
TABLE 2 | Characteristics of studies that established cutoff scores for the MMSE and MoCA in adults with low education.

\begin{tabular}{|c|c|c|c|c|c|c|c|c|}
\hline Authors, Year & Country & $\begin{array}{l}\text { Study } \\
\text { Design }\end{array}$ & $\begin{array}{l}\text { Primary Study } \\
\text { Objective }\end{array}$ & Level of Education & Sample Size & Participant Age & $\begin{array}{l}\text { Cognitive } \\
\text { Assessment Tool } \\
\text { used }\end{array}$ & Main Results \\
\hline $\begin{array}{l}\text { Chen et al., } \\
2010 \text { (25) }\end{array}$ & China & $\begin{array}{l}\text { Cross- } \\
\text { sectional }\end{array}$ & $\begin{array}{l}\text { To validate SIS for } \\
\text { quick detection of } \\
\text { cognitive impairment }\end{array}$ & $\begin{array}{l}\text { Illiterate } 7.2 \% 1-6 \\
\text { years } 16.4 \%>6 \text { years } \\
76.3 \%\end{array}$ & $\begin{array}{l}1,976 \text { (healthy } \\
\text { aging } 475, \mathrm{MCl} \\
440, \mathrm{AD} 1,061 \text { ) }\end{array}$ & $\begin{array}{l}\text { Mean age }(\mathrm{SD}) 71.87 \\
\text { years }(8.71)\end{array}$ & SIS, MMSE & 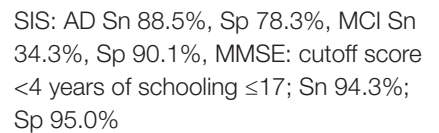 \\
\hline $\begin{array}{l}\text { Javadi PSHS } \\
\text { et al., } 2015 \text { (31) }\end{array}$ & Iran & $\begin{array}{l}\text { Cross- } \\
\text { sectional }\end{array}$ & $\begin{array}{l}\text { To characterize } \\
\text { illiterate and literate } \\
\text { older adults; PEACE } \\
\text { scale cutoff scores } \\
\text { for } A D\end{array}$ & $\begin{array}{l}\text { Different levels of } \\
\text { education-illiterate } \\
\text { and literate }\end{array}$ & $\begin{array}{l}101 \text { (controls } \\
33, \mathrm{MCl} 30 \\
\mathrm{AD} 38 \text { ) }\end{array}$ & $\begin{array}{l}\text { Mean age }(S D) A D \\
74.60 \text { years }(8.02) \\
\text { MCI } 72.5 \text { years }(7.2) \\
\text { Controls } 67.84 \text { years } \\
(7.29)\end{array}$ & $\begin{array}{l}\text { PEACE, GPCOG, } \\
\text { FAST, MMSE, WMS }\end{array}$ & $\begin{array}{l}\text { MMSE < } 4 \text { years of schooling MCl } 18.75 \\
\text { (1.75) AD } 12.64 \text { (3.78) PEACE AD } 67.5 \\
(\text { Sn } 75.8 \%, \text { Sp } 97.4 \%)\end{array}$ \\
\hline $\begin{array}{l}\text { Afgin AE et al., } \\
2012(36)\end{array}$ & Israel & $\begin{array}{l}\text { Cross- } \\
\text { sectional }\end{array}$ & $\begin{array}{l}\text { To assess prevalence } \\
\text { of } \mathrm{MCl} \text { and } \mathrm{AD} \text { and } \\
\text { conversion rate from } \\
\mathrm{MCl} \text { to } \mathrm{AD} \text { within a } \\
\text { year or more }\end{array}$ & $\begin{array}{l}0 \text { years } 51 \% 1-4 \\
\text { years } 23 \% 5-8 \text { years } \\
21 \%>8 \text { years } 5 \%\end{array}$ & $\begin{array}{l}944 \text { (controls } \\
497, \mathrm{MCl} 303 \\
\text { SD 13, VD 39, } \\
\text { AD 92) }\end{array}$ & $\begin{array}{l}\text { Mean age (years) (SD) } \\
\text { DA } 78.5(7.7) \mathrm{MCl} \\
72.8(6.1) \text { Controls } \\
70.7(5.5) \text { by age } \\
\text { groups (\%) } 65-69 \\
(39.4 \%) 70-79 \\
(46.4 \%) \geq 80(14.1 \%)\end{array}$ & $\begin{array}{l}\text { MMSE Arabic version, } \\
\text { Brookdale Cognitive } \\
\text { Screening Test }\end{array}$ & $\begin{array}{l}\text { Prevalence AD 9.8\%, MCI 32.1\% MMSE } \\
<4 \text { years of schooling cutoff scores (SD) } \\
\text { MCl illiterate } 17.8(1.9), 1-4 \text { years of } \\
\text { schooling } 19.6 \text { (3.4); AD illiterate } 12.7 \\
\text { (3.7), 1-4 years of schooling } 12.6 \text { (6.7) }\end{array}$ \\
\hline $\begin{array}{l}\text { Custodio N } \\
\text { et al., } 2017 \text { (38) }\end{array}$ & Peru & $\begin{array}{l}\text { Cross- } \\
\text { sectional }\end{array}$ & $\begin{array}{l}\text { MAT performance to } \\
\text { discriminate controls, } \\
\mathrm{MCl} \text { and AD in adults } \\
\text { with low education }\end{array}$ & $\begin{array}{l}\text { Mean schooling }(\mathrm{SD}) \\
\text { AD } 2.65 \text { years }(1.28) \\
\text { MCl } 2.53 \text { years }(1.46) \\
\text { Controls } 2.57 \text { years } \\
(1.45)\end{array}$ & $\begin{array}{l}247 \text { (controls } \\
121, \mathrm{AD} 81 \\
\mathrm{MCl} 45)\end{array}$ & $\begin{array}{l}\text { Mean age }(S D) A D \\
74.18 \text { years }(3.81) \\
\text { MCl } 71.09 \text { years } \\
(4.20) \text { Controls } 69.53 \\
\text { years }(4.11)\end{array}$ & $\begin{array}{l}\text { MAT, RAVLT, WMS, } \\
\text { TMT A and B, ROCF, } \\
\text { BNT, WCST, DS, } \\
\text { WAIS, MMSE }\end{array}$ & $\begin{array}{l}\text { MAT AD vs. MCI AUC 99.60\% MCI vs. } \\
\text { controls AUC 99.56\% Mean MMSE } \\
\text { scores (SD): AD } 18.32 \text { (2.78); MCI } 21.36 \\
\text { (0.98) MAT AD } 17.54 \text { (4.67); MCl } 30.53 \\
\text { (2.54) }\end{array}$ \\
\hline $\begin{array}{l}\text { Julayanont P. } \\
\text { et al., } 2015 \text { (45) }\end{array}$ & Thailand & $\begin{array}{l}\text { Cross- } \\
\text { sectional }\end{array}$ & $\begin{array}{l}\text { To assess validity of } \\
\text { MoCA-B for } \mathrm{MCl} \text { in a } \\
\text { population with low } \\
\text { education }\end{array}$ & $\begin{array}{l}\text { Mean schooling (SD) } \\
\text { Controls } 3.6 \text { years } \\
(1.1) \mathrm{MCl} 2.9 \text { years } \\
(1.7)\end{array}$ & $\begin{array}{l}85 \text { (controls 43, } \\
\mathrm{MCl} 42 \text { ) }\end{array}$ & $\begin{array}{l}\text { Mean age (SD) } \\
\text { Controls } 66.6 \text { years } \\
(6.7) \mathrm{MCl} 70.2 \text { years } \\
(6.6)\end{array}$ & $\begin{array}{l}\text { MoCA-B, MMSE Thai } \\
\text { version }\end{array}$ & $\begin{array}{l}\text { MMSE <4 years of schooling-illiterate } \\
\text { Mean score (SD) MMSE MCl } 18.9 \text { (3.0) } \\
\text { MoCA-B } 21.3(3.8)\end{array}$ \\
\hline $\begin{array}{l}\text { Kurt P et al., } \\
2014 \text { (48) }\end{array}$ & Turkey & $\begin{array}{l}\text { Cross- } \\
\text { sectional }\end{array}$ & $\begin{array}{l}\text { To develop a } \\
\text { composite score for } \\
\text { DEKOD in an older } \\
\text { population }\end{array}$ & $\begin{array}{l}<4 \text { years of schooling } \\
45.7 \%>5 \text { years of } \\
\text { schooling } 54.3 \%\end{array}$ & $\begin{array}{l}444 \text { (controls } \\
338, \text { dementia } \\
53, \mathrm{MCl} 53 \text { ) }\end{array}$ & $\begin{array}{l}\text { Mean age (SD) } \\
\text { controls } 70.7 \text { years } \\
\text { (5.4) dementia } 74 \\
\text { years }(7.8) \mathrm{MCl} 71.7 \\
\text { years (5.6) }\end{array}$ & $\begin{array}{l}\text { DEKOD, MMSE } \\
\text { Turkish version }\end{array}$ & $\begin{array}{l}\text { MMSE }<4 \text { years of schooling: cutoff } \\
\text { score/Sn/Sp Dementia 17-18/95\%/83\% } \\
\text { MCI 22-23/67\%/55\% DEKOD Dementia } \\
\text { 49-50/91\%/90\% MCl 60-61/70\%/65\% }\end{array}$ \\
\hline $\begin{array}{l}\text { Chen K et al., } \\
2016(55)\end{array}$ & China & $\begin{array}{l}\text { Cross- } \\
\text { sectional }\end{array}$ & $\begin{array}{l}\text { To assess } \\
\text { performance of } \\
\text { MoCA-B Chinese } \\
\text { version for screening } \\
\mathrm{MCl} \text { in older adults }\end{array}$ & $\begin{array}{l}\text { Groups by years of } \\
\text { schooling }(<6,7-12 \text {, } \\
>12) \text {. Mean schooling } \\
\text { (SD) of those with }<6 \\
\text { years: controls } 4.8 \\
\text { years (1.7), MCI } 3.3 \\
\text { years (2.4), AD } 3.7 \\
\text { years (2.5) }\end{array}$ & $\begin{array}{l}704(\mathrm{MCl} \\
264, \mathrm{AD} 160 \\
\text { controls 280) }\end{array}$ & $\begin{array}{l}\text { Mean age }(\mathrm{SD}) \text { of } \\
\text { those with }<6 \text { years } \\
\text { of schooling controls } \\
68.2 \text { years }(9.1) \mathrm{MCl} \\
68.5 \text { years }(8.5) \mathrm{AD} \\
67.9 \text { years }(9.4)\end{array}$ & $\begin{array}{l}\text { MMSE, AVLT, ROCF, } \\
\text { BNT, Verbal Fluency, } \\
\text { TMT, SCWIT, SDMT, } \\
\text { MoCA-B Chinese } \\
\text { version }\end{array}$ & $\begin{array}{l}\text { Cutoff scores } \mathrm{Sn} / \mathrm{Sp} \text { of those with }<6 \\
\text { years of schooling: MCI MoCA-BC } \\
\text { 19/87.9/81.0 MMSE 26/86.2/60.3 }\end{array}$ \\
\hline
\end{tabular}

SIS, Six-Item Screener; MMSE, Mini-Mental State Examination; PEACE, Persian Test of Elderly for Assessment of Cognition and Executive function; GPCOG, General Practitioner assessment of Cognition; FAST, Functional Assessment Staging; WMS, Wechsler Memory scale; MAT, Memory Alteration Test; RAVLT, Rey-Auditory Verbal Learning Test; TMT, Trail Making Test; BNT, Boston Naming Test; ROCF, Rey-Osterrieth Complex Figure; WCST, Wisconsin Card Sorting Test; DS, Digit Span; WAIS, Wechsler Adult Intelligence Scale-Revised; MoCA, Montreal Cognitive Assessment; MoCA B, MoCA Basic; DEKOD, Dokuz Eylül Kognitif Degerlendirme/Dokuz Eylul Cognitive Assessment; SCWIT, Stroop Color-Word Interference Test; AUC, Area Under the ROC Curve; AVLT, Auditory Verbal Learning Test; SDMT, Symbol Digit Modalities Test. 
of assessing cognitive function in individuals with low education (13). Individuals with low levels of education were excluded from many studies because there are no cutoff scores established for several assessment tools. Literacy acquisition increases performance in certain cognitive domains such as executive functions (14), improves visual processing, changes phonological codes, and strengthens the link between phonemic and graphic representation (62) However, to measure literacy through the number of years of formal education is not the most effective approach since there are so many different ways of learning even without attending school $(12,62)$.

This review study has some limitations that deserve mention. First, no information was available on the diagnostic accuracy of cognitive tools for MCI and AD. Furthermore, little information was available on the diagnostic accuracy of tools for assessing different MCI subtypes and stages of AD. Another caveat is that our search was limited to cognitive assessment tools that require a trained examiner and excluded self-administered and webbased screening tools. Besides, there is no consensus about the definition of low levels of education, which may partly explain heterogeneous results of the cognitive batteries evaluated. Lastly, the studies included this review applied various diagnostic criteria for $\mathrm{AD}$, which prevented comparisons of results across them.

\section{CONCLUSIONS}

The use of cognitive assessment tools that are easy to administer is still challenging given the high prevalence of low education in the global population. This review provides an overview of the

\section{REFERENCES}

1. Prince M, Wilmo A, Guerchet M, Ali GC, Wu YT, Prina M. World Alzheimer Report 2015: The Global Impact of Dementia an analysis of prevalence, incidence, cost and trends. London: Alzheimer's Disease International (2015). p. 87. Available from: https://www.alz.co.uk/research/ WorldAlzheimerReport2015.pdf (Access in )

2. Petersen RC. Mild cognitive impairment as a diagnostic entity. J Intern Med (2004) 256(3):183-94. doi: 10.1111/j.1365-2796.2004.01388.x

3. Ding D, Zhao Q, Guo Q, Meng H, Wang B, Luo J, et al. Prevalence of mild cognitive impairment in an urban community in china: a cross-sectional analysis of the shanghai aging study. Alzheimers Dement (2015) 11(3):300-9. e2. doi: 10.1016/j.jalz.2013.11.002

4. Petersen RC, Lopez O, Armstrong MJ, Getchius TSD, Ganguli M, Gloss D, et al. Practice guideline update summary: mild cognitive impairment Report of the Guideline Development, Dissemination, and Implementation, Subcommittee of the American Academy of Neurology. Neurol (2018) 90(3):126-35. doi: 10.1212/WNL.0000000000004826

5. Schneider JA, Arvanitakis Z, Leurgans SE, Bennett DA. The Neuropathology of Probable Alzheimer Disease and Mild Cognitive Impairment. Ann Neurol (2009) 66(2):200-8. doi: 10.1002/ana.21706

6. Handels RLH, Vos SJB, Kramberger MG, Jelic V, Blennow K, et al. Predicting progression to dementia in persons with mild cognitive impairment using cerebrospinal fluid markers. Alzheimers Dement (2017) 13(8):903-12. doi: 10.1016/j.jalz.2016.12.015

7. Studart Neto A, Nitrini R. Avaliação cognitiva à beira do leito. In: Frota NAF, Siqueira Neto JI, Balthazar MLF, Nitrini R, editors. Neurologia Cognitiva e do most commonly used instruments for cognitive screening. We found that a small number of studies evaluated adults with 4 years of formal education or less and a wide range of cutoff scores for various cognitive test batteries. Our findings further support the need for the development of specific tools for assessing illiterate adults. Cognitive ability, formal logic, and abstract reasoning should be assessed as they could provide more accurate input for screening and interpretation of cognitive tests in older adults who are either illiterate or with low levels of education. Low-cost test batteries that are easy to administer should be validated because they can make a significant impact on screening of cognitive syndromes and enable early therapeutic interventions aimed at reducing morbidity and mortality of dementia. Further studies of test batteries adjusted to larger groups of adults with low levels of education and specific MCI subtypes and AD stages could help shed light on these points.

\section{AUTHOR CONTRIBUTIONS}

Conception and design of work: JT-J, AS, GA and PB-N. Acquisition, analysis or interpretation of data and work: JT-J, AS, PB-N and GA. Drafting the work: JT-J, AS, GA, JB, JS-N and $\mathrm{PB}-\mathrm{N}$. All authors were involved in critical revision of the manuscript for important intellectual content.

\section{ACKNOWLEDGMENTS}

We thank the study participants and their families for their collaboration and support.

Envelhecimento: do conhecimento básico à abordagem clínica. OmniFarma: São Paulo (2016). p. 433.

8. Orsini A, Pezzuti G, Hulbert S. Beyond the floor effect on the Wechsler Intelligence Scale for Children - 4th Ed.(WISC-IV): calculating IQ and Indexes of subjects presenting a floored pattern of results. J Intellect Disabil Res (2015) 59(5):468-73. doi: 10.1111/jir.12150

9. Dean K, Walker Z, Jenkinson C. Data quality, floor and ceiling effects and test - retest reliability of the Mild Cognitive Impairment Questionnaire. Patient Relat Outcome Meas (2018) 9:43-7. doi: 10.2147/PROM.S145676

10. Everitt BS, Skrondal A. The Cambridge dictionary of statitistics. New York: Cambridge University Press (2010). p. 480.

11. Unesco Institute for Lifelong Learning. 3rd global report on adult learning and education: the impact of adult learning and education on health and wellbeing, employment and the labour market, and social, civic and community life. Germany: Unesco. (2016), p. 18. Available from: https://unesdoc.unesco. org/ark:/48223/pf0000245917 (Access in September 05, 2019)

12. Tucker-Drob EM, Johnson KE, Jones RN. The cognitive reserve hypothesis: A longitudinal examination of age-associated declines in reasoning and processing speed. Dev Psychol [Internet] (2009) 45(2):431-46. doi: 10.1037/a0014012

13. Mortimer JA, Snowdon DA, Markesbery WR. Head circumference, education and risk of dementia : findings from the nun study. J Clin Exp Neuropsychol (2003) 25(5):671-9. doi: 10.1076/jcen.25.5.671.14584

14. Kosmidis $\mathrm{MH}$, Zafiri M, Politimou N. Literacy versus formal schooling: influence on working memory. Arch Clin Neuropsychol (2011) 26:575-82. doi: 10.1093/arclin/acr063

15. Da Silva CG, Petersson KM, Faísca G, Ingvar H, Reis A. The effects of literacy and education on the quantitative and qualitative aspects of semantic 
verbal fluency. J Clin Exp Neuropsychol (2004) 26(2):266-77. doi: 10.1076/ jcen.26.2.266.28089

16. Wajman JR, Oliveira FF, Schultz RR, Marin SMC, Bertolucci PHF. Educational bias in the assessment of severe dementia: Brazilian cutoffs for severe Mini-Mental State Examination. Arq. Neuro-Psiquiatr (2014) 72(4):273-77. doi: 10.1590/0004-282X20140002

17. Scazufca M, Almeida OP, Vallada HP, Tasse WA, Menezes PR. Limitations of the Mini-Mental State Examination for screening dementia in a community with low socioeconomic status: results from the Sao Paulo Ageing \& Health Study. Eur Arch Psychiatry Clin Neurosci (2009) 259(1):8-15. doi: 10.1007/ s00406-008-0827-6

18. Whittemore R, Knafl K. The integrative review : updated methodology. J $A d v$ Nurs (2005) 52(5):546-53. doi: 10.1111/j.1365-2648.2005.03621.x

19. Mendes KDS, Silveira RCCP, Galvão CM. Revisão integrativa: método de pesquisa para a incorporação de evidências na saúde e na enfermagem. Texto Contexto - enferm (2008) 17(4):758-64. doi: 10.1590/ S0104-07072008000400018

20. Jackson GB. Methods for Integrative Reviews. Rev Educ Res Fall (1980) 50(3):438-60. doi: 10.3102/00346543050003438

21. Whittemore R, Knafl K. The integrative review: updated methodology. J $A d v$ Nurs (2005) 52(5):546-53. doi: 10.1111/j.1365-2648.2005.03621.x

22. Fuchs SC, Paim BS. Revisão Sistemática de Estudos Observacionais com Metanálise. Clinical \& Biomedical Research (2010) 30(3). Available at: https:// seer.ufrgs.br/hcpa/article/view/16551.

23. Sun Y, Lee HJ, Yang SC, Chen TF, Lin KN, Lin CC, et al. A Nationwide Survey of Mild Cognitive Impairment and Dementia, Including Very Mild Dementia, in Taiwan. PloS One (2014) 9(6):e100303. doi: 10.1371/journal.pone.0100303

24. Kim KW, Park JH, Kim MH, Kim MD, Kim BJ, Kim SK, et al. A nationwide survey on the prevalence of dementia and mild cognitive impairment in South Korea. J Alzheimers Dis (2011) 23(2):281-91. doi: 10.3233/JAD-2010-101221

25. Chen MR, Guo QH, Cao XY, Hong Z, Liu XH. A preliminary study of the Six-Item Screener in detecting cognitive impairment. Neurosci Bull (2010) 26(4):317-21. doi: 10.1007/s12264-010-0106-1

26. Chang J, Tse CS, Leung GT, Fung AW, Hua KT, Chiu HF, et al. Bias in discriminating very mild dementia for older adults with different levels of education in Hong Kong. Int Psychogeriatr (2014) 26(6):995-1010. doi: 10.1017/S1041610214000234

27. O'Bryant SE, Johnson G, Balldin V, Edwards M, Barber R, Williams B, et al. Characterization of Mexican Americans with Mild Cognitive Impairment and Alzheimer's Disease. J Alzheimers Dis (2013) 33(2):373-9. doi: 10.3233/ JAD-2012-121420

28. De Paula JJ, Bertola L, Ávila RT, Moreira L, Coutinho G, de Moraes EN, et al. Clinical Applicability and Cutoff Values for an Unstructured Neuropsychological Assessment Protocol for Older Adults with Low Formal Education. PloS One (2013) 8(9):e73167. doi: 10.1371/journal.pone.0073167

29. Sánchez-Benevides G, Peña-Casanova J, Casals-Coll M, Gramunt N, Molinuevo JL, Gómes-Ansón B, et al. Cognitive and Neuroimaging Profiles in Mild Cognitive Impairment and Alzheimer's Disease: Data from the Spanish Multicenter Normative Studies (NEURONORMA Project). J Alzheimers Dis (2014) 41(3):887-901. doi: 10.3233/JAD-132186

30. Mellor D, Lewis M, McCabe M, Byrne L, Wang T, Wang J, et al. Determining Appropriate Screening Tools and Cut-Points for Cognitive Impairment in an Elderly Chinese Sample. Psychol Assess (2016) 28(11):1345-53. doi: 10.1037/ pas0000271

31. Javadi PSHS, Zendehbad A, Darabi F, Khosravifar S, Noroozian M. Development and implementation of Persian test of Elderly for Assessment of Cognition and Executive function (PEACE). Electron Physician (2015) 7(7):1549-56. doi: 10.19082/1549

32. Chong MS, Lim WS, Chan SP, Feng L, Niti M, Yap P, et al. Diagnostic Performance of the Chinese Frontal Assessment Battery in Early Cognitive Impairment in an Asian Population. Dement Geriatr Cognit Disord (2010) 30(6):525-32. doi: 10.1159/000321665

33. Ng A, Chew I, Narasimhalu K, Kandiah N. Effectiveness of Montreal Cognitive Assessment for the diagnosis of mild cognitive impairment and mild Alzheimer's disease in Singapore. Singapore Med J (2013) 54(11):616-9. doi: 10.11622/smedj.2013220

34. Saka E, Mihci E, Topcuoglu MA, Balcânica S. Enhanced cued recall has a high utility as a screening test in the diagnosis of Alzheimer's disease and mild cognitive impairment in Turkish people. Arch Clin Neuropsychol (2006) 21(7):745-51. doi: 10.1016/j.acn.2006.08.007

35. Tognoni G, Ceravolo R, Nucciarone B, Bianchi F, Dell'Agnello G, Ghicopulos I, et al. From mild cognitive impairment to dementia: a prevalence study in a district of Tuscany, Italy. Acta Neurol Scand (2005) 112(2):65-71. doi: 10.1111/j.1600-0404.2005.00444.x

36. Afgin AE, Massarwa H, Schechtman E, Israeli-Korn SD, Strugatsky R, Abuful A, et al. High Prevalence of Mild Cognitive Impairment and Alzheimer's Disease in Arabic Villages in Northern Israel: Impact of Gender and Education. J Alzheimers Dis (2012) 29(2):431-9. doi: 10.3233/ JAD-2011-111667

37. Chaves ML, Camozzato AL, Godinho C, Piazenski I, Kaye J. Incidence of Mild Cognitive Impairment and Alzheimer Disease in Southern Brazil. J Geriatr Psychiatry Neurol (2009) 22(3):181-7. doi: 10.1177/0891988709332942

38. Custódio N, Lira D, Herrera-Perez E, Montesinos R, Castro-Suarez S, Cuenca-Alfaro J, et al. Memory Alteration Test to Detect Amnestic Mild Cognitive Impairment and Early Alzheimer's Dementia in Population with Low Educational Level. Front Aging Neurosci (2017) 9:278. doi: 10.3389/ fnagi.2017.00278

39. Ravaglia G, Forti P, Montesi F, Lucicesare A, Pisacane N, Rietti E, et al. Mild Cognitive Impairment: Epidemiology and Dementia Risk in an Elderly Italian Population. J Am Geriatr Soc (2008) 56(1):51-8. doi: 10.1111/j.1532-5415.2007.01503.x

40. Rahman TTA, Gaafary MME. Montreal Cognitive Assessment Arabic version: Reliability and validity prevalence of mild cognitive impairment among elderly attending geriatric clubs in Cairo. Geriatr Gerontol Int (2009) 9(1):54-61. doi: 10.1111/j.1447-0594.2008.00509.x

41. Freitas S, Simões MR, Alves G, Santana I. Montreal Cognitive Assessment Validation Study for Mild Cognitive Impairment and Alzheimer Disease. Alzheimer Dis Assoc Disord (2013) 27(1):37-43. doi: 10.1097/ WAD.0b013e3182420bfe

42. Borson S, Scanlan JM, Watanabe J, Tu SP, Lessig M. Simplifying Detection of Cognitive Impairment: Comparison of the Mini-Cog and Mini-Mental State Examination in a Multiethnic Sample. J Am Geriatr Soc (2005) 53(5):871-4. doi: $10.1111 / j .1532-5415.2005 .53269 . x$

43. Boycheva E, Contador I, Fernández-Calvo B, Ramos-Campos F, PuertasMartín V, Villarejo-Galende A, et al. Spanish version of the Mattis Dementia Rating Scale-2 for early detection of Alzheimer's disease and mild cognitive impairment. Int J Geriatr Psychiatry (2018) 33(6):832-40. doi: 10.1002/ gps.4707

44. Pezzotti P, Scalmana S, Mastromattei A, Di Lallo D. for the "Progetto Alzheimer" Working Group. The accuracy of the MMSE in detecting cognitive impairment when administered by general practitioners: A prospective observational study. BMC Fam Pract (2008) 9:29. doi: 10.1186/1471-2296-9-29

45. Julayanont P, Tangwongchai S, Hemrungrojn S, Tunvirachaisakul C, Phanthumchinda K, Hongsawat J, et al. The Montreal Cognitive Assessment-Basic: A Screening Tool for Mild Cognitive Impairment in Illiterate and Low-Educated Elderly Adults. J Am Geriatr Soc (2015) 63(12):2550-54. doi: 10.1111/jgs.13820

46. Matías-Guiu JA, Curiel RE, Rognoni T, Valles-Salgado M, FernándezMatarrubia M, Hariramani R, et al. Validation of the Spanish Version of the LASSI-L for Diagnosing Mild Cognitive Impairment and Alzheimer's Disease. J Alzheimers Dis (2017) 56(2):733-42. doi: 10.3233/JAD-160866

47. Chu LW, Ng KHY, Law ACK, Lee AM, Kwan F. Validity of the Cantonese Chinese Montreal Cognitive Assessment in Southern Chinese. Geriatr Gerontol Int (2015) 15(1):96-103. doi: 10.1111/ggi.12237

48. Kurt P, Keskinoglu P, Yaka E, Uçku R, Yener G. A composite score for Dokuz Eylul Cognitive state neurocognitivetest battery: a door-to-door survey study with illiterate, low and high educated elderly in Turkey. Turk J Geriatr (2013) 17(2):143-51. Available at: https://www.researchgate.net/ publication/288575275.

49. Bae JB, Kim YJ, Han JW, Kim TH, Park JH, Lee SB. Incidence of and Risk Factors for Alzheimer's Disease and Mild Cognitive Impairment in Korean Elderly. Dement Geriatr Cognit Disord (2015) 39(1-2):105-15. doi: $10.1159 / 000366555$

50. Paddick SM, Kisoli A, Samuel M, Higginson J, Gray WK, Dotchin CL, et al. Mild Cognitive Impairment in Rural Tanzania: Prevalence, Profile, and 
Outcomes at 4-Year Follow-up. Am J Geriatr Psychiatry (2015) 23(9):950-9. doi: 10.1016/j.jagp.2014.12.005

51. Choi SJ, Jung SS, You Y, Shin BS, Kim JE, Yoom SW, et al. Prevalence of Alzheimer's Dementia and its risk factors in community dwelling elderly Koreans. Psychiatry Invest (2008) 5(2):78-85. doi: 10.4306/pi.2008.5.2.78

52. Gavrila D, Antúnez C, Tormo MJ, Carles R, García Santos JM, Parrilla G, et al. Prevalence of dementia and cognitive impairment in Southeastern Spain: the Ariadna study. Acta Neurol Scand (2009) 120(5):300-7. doi: 10.1111/j.1600-0404.2009.01283.x

53. Wang B, Guo QH, Chen J, Zhao QH, Zhou Y, Hong Z. The clinical characteristics of 2,789 consecutive patients in a memory clinic in China. J Clin Neurosci (2011) 18(11):1473-7. doi: 10.1016/j.jocn.2011.05.003

54. Zhou Y, Ortiz F, Nuñez C, Elashoff D, Woo E, Apostolova LG, et al. Use of the MoCA in Detecting Early Alzheimer's Disease in a Spanish-Speaking Population with Varied Levels of Education. Dement Geriatr Cognit Disord (2015) 5:85-95. doi: 10.1159/000365506

55. Chen KL, Xu Y, Chu AQ, Ding D, Liang XN, Nasreddine ZS, et al. Validation of the Chinese Version of Montreal Cognitive Assessment Basic for Screening Mild Cognitive Impairment. J Am Geriatr Soc (2016) 64(12):e285-90. doi: 10.1111 /jgs. 14530

56. Matias-Guiu JA, Valles-Salgado H, Rognoni T, Hamre-Gil F, MorenoRamos T, Matias-Guiu J. Comparative Diagnostic Accuracy of the ACE-III, MIS, MMSE, MoCA, and RUDAS for Screening of Alzheimer Disease. Dement Geriatr Cognit Disord (2017) 43(5-6):237-46. doi: 10.1159/000469658

57. Huang YY, Qian SX, Guan QB, Chen KL, Zhao QH, Lu JH, et al. Comparative study of two Chinese versions of Montreal Cognitive Assessment for Screening of Mild Cognitive Impairment. Appl Neuropsychol Adult (2019), 1-6. doi: 10.1080/23279095.2019.1602530

58. Khedr EMH, Fawi G, Abbas MAA, Mohammed TAA, El-Fetoh NA, Attar GSA, et al. Prevalence of Mild Cognitive Impairmentand Dementia among the Elderly Population of Qena Governorate, Upper Egypt: A CommunityBased Study. J Alzheimers Dis (2015) 45(1):117-26. doi: 10.3233/jad-142655

59. Folstein MF, Folstein SE, McHugh PR. Mini-mental state: a practical method for grading the cognitive state of patients for the clinician. J Psychiatr Res (1975) 12(3):189-98. doi: 10.1016/0022-3956(75)90026-6

60. Nasreddine ZS, Phillips NA, Bédirian V, Charbonneau S, Whitehead V, Collin I, et al. The Montreal Cognitive Assessment, MoCA: a brief screening tool for Mild Cognitive Impairment. J Am Geriatr Soc (2005) 53(4):695-9. doi: $10.1111 / j .1532-5415.2005 .53221 . x$
61. Roe CM, Xiong C, Miller JP, Morris JC. Education and Alzheimer disease without dementia: support for the cognitive reserve hypothesis. Neurol (2007) 68(3):223-8. doi: 10.1212/01.wnl.00002513 03.50459.8a

62. Dehaene S, Cohen L, Morais J, Kolinsky R. Illiterate to literate: behavioural and cerebral changes induced by reading acquisition. Nat Rev Neurosci (2015) 16(4):234-44. doi: 10.1038/nrn3924

63. Marques P, Soares JM, Magalhães R, Santos NC. Sousa N. The bounds of education in the human brain connectome. Sci Rep (2015) 5:12812. doi: $10.1038 /$ srep 12812

64. De Roeck EE, Engelborghs S, Dierckx E. Next generation brain health depends on early Alzheimer disease diagnosis: from a timely diagnosis to future population screening. J Am Med Dir Assoc (2016) 17:452-3. doi: 10.1016/j.jamda.2016.02.015

65. De Roeck EE, De Deyn PP, Dierckx E, Engelborghs S. Brief cognitive screening instruments for early detection of Alzheimer's disease: a systematic review. Alzheimers Res Ther (2019) 11(1):21. doi: 10.1186/s13195-019-0474-3

66. Mitchell AJ. A meta-analysis of the accuracy of the mini-mental state examination in the detection of dementia and mild cognitive impairment. J Psychiatr Res (2009) 43(4):411-31. doi: 10.1016/j.jpsychires.2008.04.014

67. Brucki SMD, Nitrini R, Caramelli P, Bertolucci PHF, Okamoto IH. Sugestões para o uso do mini-exame do estado mental no Brasil. Arq Neuro-psiquiatr (2003) 61(3B):777-81. doi: 10.1590/S0004-282X2003000500014

68. Julayanont P, Phillips N, Chertkow H, Nasreddine ZS. Montreal Cognitive Assessment (MoCA): Concept and clinical review. In: Larner AJ, ed. Cognitive Screening Instruments: A Practical Approach. London: Springer (2013) p. 111-51. Available from: https://www.concordia.ca/content/dam/ artsci/research/caplab/docs/Julayanontetal2013MoCAreview.pdf (Access in September)

Conflict of Interest: The authors declare that the research was conducted in the absence of any commercial or financial relationships that could be construed as a potential conflict of interest.

Copyright $\odot 2019$ Tavares-Júnior, de Souza, Alves, Bonfadini, Siqueira-Neto and Braga-Neto. This is an open-access article distributed under the terms of the Creative Commons Attribution License (CC BY). The use, distribution or reproduction in other forums is permitted, provided the original author(s) and the copyright owner $(s)$ are credited and that the original publication in this journal is cited, in accordance with accepted academic practice. No use, distribution or reproduction is permitted which does not comply with these terms. 